\title{
Disponibilidade no acervo do Portal de Periódicos CAPES dos periódicos citados em teses da Universidade Federal de Santa Catarina
}

\author{
Fabio Lorensi do Canto \\ Mestre; Universidade Federal de Santa Catarina, Florianópolis, SC, Brasil; \\ fabio.lc@ufsc.br \\ Adilson Luiz Pinto \\ Doutor; Universidade Federal de Santa Catarina, Florianópolis, SC, Brasil; \\ adilson.pinto@ufsc.br
}

\begin{abstract}
Resumo: Este trabalho tem por objetivo verificar a disponibilidade no acervo do Portal de Periódicos CAPES dos periódicos científicos citados em teses da Universidade Federal de Santa Catarina. Visa também analisar os periódicos com mais artigos citados, identificando o país de origem, a indexação nas bases de dados Web of Science, Scopus, Latindex e SciELO e a classificação Qualis. Essas análises serão realizadas comparativamente a partir das nove grandes áreas do conhecimento da CAPES, visando detectar semelhanças e diferenças entre os padrões de uso de periódicos e as necessidades informacionais dos pesquisadores doutorais. Utiliza as técnicas de amostragem estratificada uniforme e aleatória simples para definir a amostra de 90 teses, sendo dez de cada grande área do conhecimento, correspondendo a 20,3\% do total de 443 teses defendidas em 2015 na Universidade Federal de Santa Catarina. Os resultados mostram que $84,76 \%$ dos periódicos eletrônicos citados nas teses estão disponíveis no Portal, sendo Ciências Biológicas $(94,15 \%)$ a área com maior disponibilidade e Linguística, Letras e Artes (69,23\%), com menor disponibilidade. Periódicos estrangeiros, sobretudo dos Estados Unidos, da Inglaterra e da Holanda, são os mais utilizados na maioria das áreas, exceto nas Ciências Sociais, Humanas e Linguística, Letras e Artes, que utilizam mais periódicos nacionais. Do total de periódicos do núcleo, $71 \%$ está indexado na Web of Science, $82 \%$ na Scopus, $9 \%$ no Latindex e $16 \%$ no SciELO. Periódicos A1 e A2 são mais utilizados na média geral. Constata-se, portanto, que o acervo Portal de Periódicos CAPES é significativamente adequado as necessidades dos pesquisadores doutorais, principalmente nas ciências naturais, tecnológicas e da saúde, áreas que fazem maior uso de periódicos estrangeiros, indexados na Web of Science e na Scopus e de classificação Qualis A1 e A2.
\end{abstract}

Palavras-chave: Periódicos científicos. Análise de citação. Estudos métricos da informação. Portal de Periódicos Capes. 


\section{Introdução}

O aumento exponencial das atividades da ciência e da produção científica formal, expressa em livros, periódicos e outros documentos escritos, ocasionou problemas de recuperação e acesso à informação, bem como a necessidade de avaliação de inputs e outputs dos processos pertinentes à ciência. As questões relacionadas à mensuração da informação deram origem a estudos orientados para a avaliação quantitativa dos fenômenos científicos, os quais passaram a ser definidos como estudos métricos da informação.

Entre as variadas técnicas utilizadas em estudos métricos, a análise de citação é uma das mais recorrentes. Essa técnica, baseada na coleta, análise e apresentação de dados relativos à citação de documentos, possui diversas aplicações no âmbito da cienciometria e da bibliometria. Entre as principais aplicações destacam-se o impacto de publicações e de autores medido pela frequência de citações recebidas, a identificação de padrões de uso e citação de documentos por pesquisadores, análises de redes de citação e cocitação de autores e a avaliação de acervos, sejam físicos ou eletrônicos, a partir de uma relação de publicações citadas em trabalhos científicos, tais como artigos, dissertações e teses.

Nesse sentido, o presente estudo se caracteriza como de natureza bibliométrica e cienciométrica, baseado na técnica da análise de citação. Tem dupla finalidade, sendo a primeira de verificar a disponibilidade no acervo do Portal de Periódicos CAPES dos periódicos com artigos citados em teses da Universidade Federal de Santa Catarina (UFSC); e a segunda de identificar e analisar o núcleo de periódicos, ou seja, os periódicos com maior frequência de artigos citados. As variáveis investigadas nos periódicos do núcleo são o país de origem, a indexação nas bases de dados Web of Science (WoS), Scopus, Latindex e SciELO e, por fim, a classificação Qualis/Capes das publicações.

Ambas as análises - a disponibilidade do acervo do Portal e a do núcleo de periódicos - serão realizadas a partir das nove grandes áreas do conhecimento da Coordenação de Aperfeiçoamento de Pessoal de Nível Superior (CAPES), visando detectar semelhanças e diferenças entre os padrões 
de uso de periódicos e a adequação do acervo do Portal as necessidades dos pesquisadores das diferentes áreas.

Embora estudos de análise de citação sejam frequentes na literatura, o estudo em tela se diferencia justamente por enfatizar a disponibilidade do acervo do Portal a partir das diferenças nos padrões de uso de periódicos entre as grandes áreas do conhecimento. Diverge, portanto, de estudos que se dedicam a analisar de forma mais detalhada a cobertura do Portal em relação a uma área ou campo específico.

Pretende-se, outrossim, enfatizar a importância do Portal para a formação de pesquisadores de doutorado das diversas áreas do conhecimento no país, e a necessidade premente de manutenção e ampliação de seu acervo. Visa também apresentar uma visão mais detalhada dos padrões de uso e citação de periódicos pelos pesquisadores da UFSC, o que pode contribuir tanto sob o aspecto didático, no âmbito das coordenações dos programas de pós-graduação, quanto sob o aspecto do suporte à pesquisa, no âmbito da Biblioteca Universitária.

Este trabalho apresenta significativo acréscimo de conteúdo teórico e empírico em relação ao apresentado no $6^{\circ}$ Encontro Brasileiro de Bibliometria e Cientometria (6 $\left.6^{\circ} \mathrm{EBBC}\right)$ (CANTO; PINTO, 2018). Os acréscimos teóricos são a síntese histórica e os dados comparativos referentes ao crescimento do Portal de Periódicos CAPES. Sob ponto de vista empírico, foram acrescidas análises relativas à disponibilidade de artigos citados nas teses do acervo do Portal de Periódicos CAPES, bem como de indexação em bases de dados e classificação Qualis/Capes dos periódicos do núcleo.

\section{Portal de Periódicos CAPES}

O início do ano 2000 representou um período de mudanças nos processos de divulgação e acesso à ciência, considerando que o crescimento da Internet e dos formatos eletrônicos de documentos já influenciavam a produção e a comunicação científica. Neste período, observa-se um grande impacto no sistema de editoração e assinatura de periódicos científicos, bem como inúmeros 
projetos de migração do formato impresso para o eletrônico (SANTANA et al., 2012).

A partir da estrutura e a experiência em assinatura consorciada de periódicos adquirida com o $\mathrm{PROBE}^{1}$, em 11 de novembro de 2000 foi criado o Portal de Periódicos CAPES, fato que deu início a era das bibliotecas digitais para a comunidade científica brasileira (MIRANDA; CARVALHO; RAMOS, 2012; SANTANA et al., 2012).

A criação do Portal possibilitou a CAPES sistematizar o acesso à informação científica, especialmente periódicos internacionais e bases de dados referenciais. Entre os objetivos alcançados pelo programa, se destacam a redução do custo médio de acesso ao conteúdo por usuário, o acesso unificado à plataforma de busca e a resolução do problema da descontinuidade das assinaturas de periódicos impressos em bibliotecas universitárias (MIRANDA; CARVALHO; RAMOS, 2015).

Desde a sua criação, o Portal vem registrando aumento constante em seus indicadores. $\mathrm{O}$ acervo passou de 8.500 títulos de periódicos no ano de 2004 para mais de 38 mil no ano de 2017. O número de downloads de conteúdo em texto completo passou de aproximadamente 13 mil em 2004 para mais de 44 mil em 2013. Neste mesmo período, o número de instituições participantes passou de 133 para 422. O investimento financeiro aumentou de aproximadamente $\mathrm{R} \$ 48$ milhões em 2004 para mais de R 357 milhões em 2016 (CAPES, 2017).

Diante do impacto do Portal para a pesquisa científica brasileira, alguns estudos quantitativos do acervo passaram a ser realizados.

Fernandes e Cendón (2010) analisaram citações de artigos de periódicos em teses e dissertações do Programa de Pós-graduação em Ciência da Informação da Universidade Federal de Minas Gerais (PPGCI/UFMG), publicadas nos anos de 2005, 2006 e 2007, com o intuito de verificar a disponibilidade dos periódicos no acervo do Portal. Os resultados indicaram que $45 \%$ dos títulos que tiveram artigos citados na amostra estavam disponíveis no Portal, índice considerado abaixo do ideal pelos autores.

Gibrowski (2011) realizou análise quantitativa do acervo de periódicos de Ciências Agrárias do Portal, utilizando como base 152 periódicos dessa 
grande área com melhor índice no Article Influence Score do Journal Citation Reports (JCR) no ano de 2009. Constatou que 69,7\% do total de periódicos listados estavam disponíveis no Portal, sendo 81,6\% de Agronomia, 54,5\% de Agronomia Multidisciplinar, 77,8\% de Agronomia Política e Econômica, 86,7\% de Ciências do Solo, 70\% de Engenharia Agrícola e 42,9\% de Horticultura.

Mattos e Dias (2012) analisaram quantitativamente o acervo de periódicos da área de Administração do Portal. Como técnica, utilizaram a análise de citação de 85 teses defendidas no Programa de Pós-Graduação em Administração da Escola de Administração da Universidade Federal do Rio Grande do Sul (PPGA/UFRGS) entre os anos de 1999 e 2007. Eles constataram que dos 40 periódicos com maior frequência de artigos citados na amostra, 30 deles estavam disponíveis no Portal, o que corresponde a 75\%. Entre os disponíveis, contudo, havia oito títulos com alguma espécie de lacuna entre os fascículos da coleção.

Klaes (2012) conduziu estudo quantitativo do acervo de Odontologia do Portal, utilizando como base a lista de periódicos indexados em cinco bases de dados. Constatou que 88,31\% dos títulos dessa área indexados no JCR, 87,50\% na WoS, 75,49\% no Scientific Journal Rankings (SJR), 68,51\% na Scopus e 57,38\% na MEDLINE estavam disponíveis no Portal, resultando na média geral de $79,95 \%$ de disponibilidade.

Galdência e Lima (2015) avaliaram o acervo do Portal a partir de 6.519 citações de 19 teses e 43 dissertações defendidas em 2012 em nove cursos atendidos por uma biblioteca da área Biomédica e de Ciências da Saúde da Universidade Federal do Rio de Janeiro (UFRJ). Os resultados apontaram que $85 \%$ das obras citadas estavam disponíveis no acervo do Portal.

Observa-se que os estudos destinados à avaliação do acervo do Portal são delimitados a uma área ou grande área do conhecimento. Entre as áreas analisadas, verificou-se a disponibilidade de periódicos no Portal variando entre 45\% (Ciência da Informação), 69,7\% (Ciências Agrárias), 75\% (Administração) e 79,95\% (Odontologia) e 85\% (Saúde e Biomedicina). Não foi identificada, contudo, análise comparativa entre áreas, lacuna a ser preenchida por esta pesquisa. Além disso, com exceção do estudo de Galdência e Lima (2015), 
todos os demais foram realizados há mais de cinco anos, sendo que após esta data houve um aumento aproximado de 12,5\% no número de periódicos disponíveis.

\section{Estudos métricos}

Estudos métricos da informação são um conjunto de métodos e técnicas de pesquisa quantitativa da Ciência da Informação. As primeiras pesquisas métricas eram aplicadas na mensuração de aspectos relacionados à produção, disseminação e uso da informação registrada (TAGUE-SUTCLIFFE, 1992), bem como na observação de regularidades nos processos de produção e comunicação da ciência (BAILÓN-MORENO et al., 2005). Esses estudos foram posteriormente acrescidos por investigações acerca dos aspectos funcionalista e sociológico da ciência, pelo desenvolvimento de indicadores para avaliação das atividades científicas e para definição de políticas governamentais de fomento à pesquisa.

Em função de suas variadas aplicações, os estudos métricos se firmaram como campo emergente da ciência, sobretudo em áreas interdisciplinares como as Ciências Sociais (BAILÓN-MORENO et al., 2005). Dependendo da natureza das métricas aplicadas, estas podem contribuir para o processo de tomada de decisão relacionado às atividades científicas, tanto em nível de planejamento e investimento (inputs), quando em nível de avaliação de resultados (outputs) (PINTO; ELIAS; VIANNA, 2014).

Com a consolidação dos estudos métricos, foi possível observar uma ampliação das aplicações ou especificações métricas, direcionada para objetos de estudo ou fenômenos específicos. Além das tradicionais bibliometria e cienciometria, outras tipologias como a informetria (quantificação da informação recuperada) (WORMELL, 1998), a webometria (indicadores web) (GOUVEIA, 2012) e a arquivometria (métricas em arquivos) (PINTO; ELIAS; VIANNA, 2014) ganharam espaço no rol de estudos métricos relevantes.

Como disciplina científica, a bibliometria sedimentou a aplicação de análises estatísticas no tocando ao estudo das características de publicações 
científicas, tais como livros, artigos e periódicos científicos, teses e dissertações. Se aplica, nesse sentido, na análise dos aspectos relacionados à criação dos documentos, tais como autoria e coautoria, características físicas, temáticas e metodológicas. Também se aplica aos processos de mensuração do tratamento técnico de documentos em sistemas de recuperação da informação em bibliotecas, como indexação e elaboração de índices e resumos. Pode ainda estar presente na mensuração de condições inerentes ao uso e reprodução de documentos, como por exemplo, por meio de estudos de citações e de referências (SPINAK, 1998).

As chamadas leis bibliométricas, quais sejam, Bradford (análise de dispersão de publicações), Zipf (análise de terminologias e questões semânticas) e Lokta (análise de autoridades), contribuíram para a formação de uma tendência de pesquisas métricas, tornando-se os pilares não apenas da bibliometria, mas também da cienciometria (BAILÓN-MORENO et al., 2005).

A cienciometria, considerada a ciência da ciência, é um conjunto de métodos e técnicas quantitativas de coleta e análise de dados relativos à ciência enquanto atividade social e econômica. Tem origem em uma visão funcionalista e sociológica da ciência e se preocupa com a construção de indicadores com a finalidade de orientar a elaboração de políticas de fomento bem como conduzir a avaliação de resultados de atividades científicas (SPINAK, 1998).

A cienciometria possui fundamentos teóricos próprios, mas também se baseia nas leis bibliométricas de Bradford, Lotka e de Zipf para o estabelecimento de indicadores, aplicáveis não apenas na Ciência da Informação, mas também em outras áreas do conhecimento (BAILÓNMORENO et al., 2005).

Muitos indicadores, tanto bibliométricos quanto cienciométricos, têm como base a frequência de citação de documentos, que se traduzem em índices como o Fator de Impacto, o Article Influence Score, o Índice h e mesmo a classificação Qualis de periódicos, considerando que algumas áreas de avaliação incluem o fator de impacto como critério de classificação de títulos nos estratos A1 e A2. 


\section{Análise de citação}

Considerando a importância da citação na produção e na comunicação científica, inúmeros autores (GARFIELD, 1972; CRONIN, 1984, LEYDESDORFF, 1998; KAPLAN, 1965; SMALL, 2004; RIVIERA, 2013) passaram a investigar, por diferentes abordagens, as funções e as características da prática da citação na ciência mundial. A afirmação comum ao justificar a necessidade de se compreender a função da citação na comunicação científica reside na ideia de que ela seria um elemento central na (re)produção da ciência, a qual todo pesquisador incorpora no processo de elaboração de documentos. A citação se constitui, outrossim, em uma espécie de instituição com padrões e valores obrigatórios aos seus integrantes (CRONIN, 1984).

A análise de citação é um método de pesquisa quantitativo que analisa citações de periódicos, trabalhos científicos e de autores para examinar suas características e identificar padrões de uso (HOU, 2017). O elemento central desta técnica, a citação, pode ser definida como a menção a um trabalho anteriormente publicado, que pode ser expresso por meio uma entrada bibliográfica realizada em nota de rodapé, lista de referência ou bibliografia de um documento que contenha informações suficientes (autor, título, editor, ano) para identificar o item original. A análise de citação, em sua dimensão normativa (SILVEIRA, 2016), considera os atributos do documento citado e não o conteúdo de seu texto. Assim, cada citação é vista como uma unidade de dados distinta, independente de quantas vezes foi mencionada no texto citante ou se a menção ao documento citado foi positiva ou negativa (ROSEMBERG, 2015).

A primeira menção ao termo citation analysis é atribuída à Gross and Grossa em um estudo dos periódicos citados no Journal of the American Chemical Society realizado em 1927 (ROSEMBERG, 2015). Neste período, os trabalhos de Lotka e Bradford também contribuíram para criação dos fundamentos teóricos da análise de citação (HOU, 2017). A sua popularização, contudo, veio com na Década de 1960 com o desenvolvimento do Science Citation Index (FEYEREISEN; SPOIDEN, 2009). Nas décadas seguintes há 
uma ampliação das abordagens baseadas na citação com os estudos de redes de cocitação (author co-citation analysis).

Verifica-se uma tendência na aplicação da análise de citações com a finalidade de identificar padrões de citação de documentos por pesquisadores, periódicos ou instituições entre diferentes disciplinas (ENGER, 2009; ROSEMBERG, 2015; STEPHENS et al., 2013). Este tipo de análise possibilita uma visão geral da forma como cada área tende a publicar resultados de pesquisas, bem como permite observar as diferenças principais entre a estrutura delas (STRADER, 2012). Entre as variáveis investigadas incluem-se a quantidade de documentos citados por publicação, o tipo de material citado (livro, periódico, anais etc.) o formato da publicação (impresso ou eletrônico) e demais características da publicação (idioma, dada e país de origem) (HOFFMANN; DOUCETTE, 2012).

Esta técnica pode ser aplicada também para avaliação de acervos, considerando que permite a identificação das necessidades de pesquisadores enquanto usuários de sistemas de informação, como bibliotecas e bases de dados. Para essa finalidade, utiliza-se a lista de referências de um trabalho científico e compara-se com o acervo a fim de verificar a disponibilidade dos documentos citados (BECKER; CHIWARE, 2015; ROSEMBERG, 2015).

Estes trabalhos utilizados como bibliografia padrão podem ser artigos de periódicos científicos, livros, dissertações e, principalmente, teses de doutorado. A recomendação para uso de teses decorre do fato este tipo de publicação normalmente é fruto de pesquisas com avançado nível de profundidade (KUSHKOWSKI et al., 2003), resultando em uma extensa lista referências sobre o tema abordado.

O método de avaliação de acervos por meio de análise de citação é visto como complementar aos demais métodos comumente utilizados, tais como avaliação por especialista ou análise de frequência de uso (FERNANDES; CENDÓN, 2010). Possibilita uma visão mais precisa do tipo de material e a frequência com que é utilizado por pesquisadores. No caso de periódicos, é possível identificar, inclusive, os títulos com artigos mais citados, podendo se definir o núcleo de periódicos mais produtivos em cada campo de pesquisa. 
Nesse sentido, se verifica a possibilidade de aplicação da análise de citação de teses para avaliação do acervo do Portal de Periódicos CAPES, considerado o principal programa destinado a prover acesso à informação científica aos pesquisadores brasileiros.

\section{Metodologia}

Trata-se de estudo de alcance exploratório e descritivo e de natureza quantitativa. Foram utilizados os métodos de amostragem estratificada uniforme e aleatória simples (BARBETTA, 2012). A escolha deste método se deu em razão da ênfase na análise das diferenças entre as grandes áreas do conhecimento.

Foram selecionadas 90 teses, 20,3\% do universo composto por 443 teses defendidas na UFSC no ano de 2015 e descritas no catálogo da Biblioteca Universitária. Cada grande área foi representada com dez teses, sendo que todos os programas de doutorado contaram com ao menos uma tese na amostra, garantindo assim a variação temática.

A partir das teses selecionadas, utilizou-se a análise de citação para verificar a disponibilidade dos periódicos eletrônicos com artigos citados no acervo do Portal, efetuando-se a busca por título ou ISSN dos periódicos no Portal. Também foi verificado o índice de disponibilidade dos periódicos de acordo com a frequência de artigos citados.

Além disso, foi identificado também o núcleo de periódicos de cada grande área do conhecimento, ou seja, os periódicos com maior frequência de artigos citados. O núcleo foi definido com base no resultado da raiz quadrada do número de citações do periódico com mais artigos citados. Exemplo: se o periódico com maior número de artigos citados em determinada área obteve 49 citações, todos os periódicos com sete ou mais citações na área integraram o núcleo.

A partir dos periódicos listados no núcleo, foram identificados: a) o país de origem das publicações; b) o percentual de periódicos indexados na WoS, na Scopus, no Latindex e no SciELO; c) a classificação Qualis/Capes dos 
periódicos. A classificação Qualis considerada foi a mais elevada do periódico na grande área objeto de análise. Por exemplo: na grande área Ciências Exatas e da Terra foi considerada a maior classificação obtida pelo periódico nas áreas de avaliação Astronomia/Física, Ciência da Computação, Geociências, Matemática/Probabilidade, Estatística e Química. Os dados foram tabulados em planilhas Excel.

\section{Resultados e discussão}

Foram analisadas 15.342 referências nas 90 teses da amostra. Destas, 8.100 referências foram de artigos de periódicos científicos eletrônicos, número equivalente a $57,2 \%$ do total de obras citadas.

\subsection{Avaliação do acervo do Portal de Periódicos CAPES}

A Tabela 1 mostra que 84,67\% dos periódicos eletrônicos com artigos citados nas teses estavam disponíveis no acervo do Portal. As grandes áreas com maior percentual foram Ciências Exatas e da Terra e Ciências Biológicas, que tradicionalmente fazem maior uso de periódicos na produção científica em relação a outros tipos de materiais. As áreas com menor índice foram Ciências Sociais Aplicadas e Linguística, Letras e Artes, fato que pode ser atribuído a menor influência do periódico na produção científica dessas áreas, ou ainda da política de seleção de conteúdo da CAPES. As demais áreas obtiveram índice próximo ao da média geral de $84,67 \%$ de disponibilidade.

Tabela 1 - Periódicos citados nas teses da UFSC disponíveis no Portal de Periódicos CAPES, segundo a grande área de conhecimento

\begin{tabular}{lccc}
\hline \multicolumn{1}{c}{ Área } & Total de títulos & Disponíveis (títulos) & Disponíveis (\%) \\
\hline Ciências Exatas e da Terra & 342 & 322 & 94,15 \\
Ciências Biológicas & 663 & 611 & 92,16 \\
Engenharias & 275 & 228 & 82,91 \\
Ciências da Saúde & 419 & 365 & 87,11 \\
Ciências Agrárias & 414 & 351 & 84,78 \\
Ciências Sociais Aplicadas & 248 & 195 & 78,63 \\
Ciências Humanas & 235 & 202 & 85,96 \\
Linguística, Letras e Artes & 117 & 81 & 69,23 \\
Multidisciplinar & 464 & 404 & 87,07 \\
\hline Média Geral & & $\mathbf{8 4 , 6 7}$ \\
\hline
\end{tabular}

Fonte: Dados da pesquisa (2018). 
Os dados obtidos indicam um aumento qualitativo do acervo do Portal em comparação áreas que já haviam sido objeto de análise na literatura. Ciências Agrárias, que obteve $84,78 \%$ na presente análise, registrou um aumento aproximado de $21 \%$ em relação à avaliação de Gibrowski (2011), que mensurou 69,7\% de disponibilidade de títulos da mesma grande área. Ciências da Saúde $(87,11 \%)$ obteve aumento aproximado de $9 \%$ em relação ao percentual obtido por Klaes (2012), que apurou 79,95\% de disponibilidade em Odontologia, bem como aumento de 2\% em relação ao estudo de Galdência e Lima (2015). Ciências Sociais Aplicadas $(78,63 \%)$ apresentou aumento aproximado de 5\% em relação ao estudo de Mattos e Dias (2010), que mensuraram 75\% de disponibilidade de periódicos citados em teses de Administração.

Assim, considerando o lapso temporal entre a realização destas pesquisas e da presente, é possível afirmar que o crescimento do acervo do Portal refletiu nos aumentos obtidos, embora os dados utilizados como comparação tenham se limitado às referidas áreas.

No que tange à disponibilidade de periódicos em relação à frequência de citações, a Tabela 2 mostra um aumento em relação à análise por título, com índice geral de 89,21\% de citações de artigos de periódicos disponíveis no Portal. Esse aumento é decorrente da maior frequência de citação a periódicos disponíveis em comparação com os periódicos não disponíveis.

Tabela 2 - Artigos citados nas teses da UFSC disponíveis no Portal de Periódicos CAPES, segundo a grande área de conhecimento

\begin{tabular}{|c|c|c|c|}
\hline Área & $\begin{array}{l}\text { Artigos } \\
\text { citados }\end{array}$ & $\begin{array}{c}\text { Disponíveis } \\
\text { (Artigos) }\end{array}$ & $\begin{array}{c}\text { Disponíveis } \\
(\%)\end{array}$ \\
\hline Ciências Exatas e da Terra & 1032 & 1009 & 97,77 \\
\hline Ciências Biológicas & 2002 & 1926 & 96,20 \\
\hline Engenharias & 624 & 555 & 88,94 \\
\hline Ciências da Saúde & 1151 & 1061 & 92,18 \\
\hline Ciências Agrárias & 1219 & 1123 & 92,12 \\
\hline Ciências Sociais Aplicadas & 407 & 344 & 84,52 \\
\hline Ciências Humanas & 406 & 360 & 88,67 \\
\hline Linguística, Letras e Artes & 157 & 111 & 70,70 \\
\hline Multidisciplinar & 1102 & 1011 & 91,74 \\
\hline Média Geral & & & 89,21 \\
\hline
\end{tabular}


Os índices obtidos nesta variável indicam que o incremento do número de periódicos nos últimos anos tem reflexo no desempenho nas avaliações quantitativas do acervo, mesmo que as metodologias das pesquisas utilizadas na comparação não tenham sido as mesmas.

\subsection{Avaliação dos periódicos do núcleo}

A Tabela 3 mostra a relação (total e percentual) de periódicos e de artigos do núcleo. Observa-se que parte substancial de citações a artigos se concentram em um pequeno percentual de títulos. Essa concentração, na maioria das áreas, é em percentual próximo ou inferior a $10 \%$ do total de periódicos, que são responsáveis pela publicação de aproximadamente $40 \%$ do total de artigos citados.

Tabela 3 - Núcleo de periódicos citados nas teses da UFSC, segundo a grande área do conhecimento

\begin{tabular}{l|c|c|c|c|c|c}
\hline \multirow{2}{*}{\multicolumn{1}{c|}{ Áreas }} & \multicolumn{3}{c|}{ Periódicos } & \multicolumn{3}{c}{ Artigos } \\
\cline { 2 - 7 } & $\begin{array}{c}\text { Total } \\
\text { Amostra }\end{array}$ & Núcleo & $\%$ & $\begin{array}{c}\text { Total } \\
\text { Amostra }\end{array}$ & Núcleo & $\%$ \\
\hline Exatas e da Terra & 343 & 43 & 12,53 & 1019 & 396 & 38,86 \\
\hline Biológicas & 663 & 78 & 11,76 & 2002 & 967 & 48,3 \\
\hline Engenharias & 275 & 20 & 7,27 & 624 & 249 & 39,9 \\
\hline Saúde & 419 & 31 & 8,14 & 1151 & 499 & 43,35 \\
\hline Agrárias & 414 & 37 & 8,93 & 1219 & 603 & 49,46 \\
\hline Sociais Aplicadas & 248 & 31 & 12,5 & 407 & 164 & 41,12 \\
\hline Humanas & 235 & 11 & 4,68 & 406 & 97 & 23,89 \\
\hline Linguística & 117 & 28 & 23,93 & 157 & 68 & 43,31 \\
\hline Multidisciplinar & 464 & 32 & 6,9 & 1102 & 460 & 41,74 \\
\hline
\end{tabular}

Conforme a Tabela 4, Ciências Sociais Aplicadas, Linguística, Letras e Artes e Ciências Humanas possuem maior tendência de citação de artigos de periódicos nacionais, o que é um indício da realização de pesquisas voltadas para temas de interesse nacional e local. Esse resultado também pode ser um indicador de uso de periódicos independentes e vinculados a instituições de ensino superior nacionais. 
Tabela 4 - País de origem dos periódicos do núcleo das teses da UFSC, segundo a grande área do conhecimento

\begin{tabular}{l|c|c|c|c|c|c|c}
\hline & Brasil & EUA & Inglaterra & Holanda & Espanha & Suíça & Outros \\
\hline Exatas e da Terra & $5 \%$ & $37 \%$ & $35 \%$ & $11 \%$ & $0 \%$ & $5 \%$ & $7 \%$ \\
\hline Biológicas & $0 \%$ & $40 \%$ & $35 \%$ & $18 \%$ & $0 \%$ & $1 \%$ & $6 \%$ \\
\hline Saúde & $29 \%$ & $26 \%$ & $19 \%$ & $0 \%$ & $0 \%$ & $0 \%$ & $26 \%$ \\
\hline Engenharias & $0 \%$ & $26 \%$ & $26 \%$ & $37 \%$ & $0 \%$ & $11 \%$ & $0 \%$ \\
\hline Agrárias & $13 \%$ & $27 \%$ & $30 \%$ & $30 \%$ & $0 \%$ & $0 \%$ & $0 \%$ \\
\hline Sociais Aplicadas & $87 \%$ & $3 \%$ & $0 \%$ & $7 \%$ & $3 \%$ & $0 \%$ & $0 \%$ \\
\hline Humanas & $55 \%$ & $27 \%$ & $9 \%$ & $9 \%$ & $0 \%$ & $0 \%$ & $0 \%$ \\
\hline $\begin{array}{l}\text { Linguística, letras } \\
\text { e artes }\end{array}$ & $64 \%$ & $3 \%$ & $7 \%$ & $0 \%$ & $18 \%$ & $0 \%$ & $8 \%$ \\
\hline Multidisciplinar & $23 \%$ & $39 \%$ & $19 \%$ & $7 \%$ & $3 \%$ & $6 \%$ & $3 \%$ \\
\hline Média Geral & $31 \%$ & $25 \%$ & $20 \%$ & $13 \%$ & $3 \%$ & $3 \%$ & $5 \%$ \\
\hline
\end{tabular}

Ciências da Saúde, embora tenha um maior percentual de citação de periódicos internacionais, especialmente dos Estados Unidos e da Inglaterra, também tende a citar artigos de periódicos nacionais. Estes dados demostram que, além de uma evidência de internacionalização de pesquisas, também há um enfoque regional e nacional, voltadas, por exemplo, para incidência geográfica de enfermidades ou programas nacionais de saúde.

Por outro lado, Engenharias e Ciências Biológicas não contaram com nenhuma citação de artigos de periódicos nacionais, sendo EUA e Inglaterra as principais origens desta e Holanda daquela área. Ciências Exatas apresenta tendência semelhante a Biológicas, exceto pela incidência de periódicos nacionais na área de Exatas. Estes resultados indicam a internacionalização de pesquisas, além da evidência de uso de periódicos de editores comerciais, tais como a Elsevier, com sede na Holanda.

Como se observa da Tabela 5, todos os periódicos do núcleo das áreas de Ciências Exatas e da Terra, Ciências Biológicas, Engenharias e Ciências Agrárias são indexados na WoS e Scopus, indicando internacionalização das pesquisas dessas áreas, bem como o uso de periódicos considerados de elite ou alto impacto.

Ciências Social Aplicadas e Linguística, Letras e Artes são as áreas com menor índice de citação de periódicos indexados na WoS e Scopus. Sociais Aplicadas e Ciências da Saúde são as áreas com maior número de periódicos indexados na SciELO e no Redalyc, indicando uma tendência de pesquisa de temas de interesse nacional (Brasil) e regional (América Latina). 
Tabela 5 - Periódicos do núcleo das teses da UFSC indexados em bases de dados, segundo a grande área do conhecimento

\begin{tabular}{l|c|c|c|c}
\hline & WoS & Scopus & Redalic & SciELO \\
\hline Ciências Exatas e da Terra & $100 \%$ & $100 \%$ & $0 \%$ & $8 \%$ \\
\hline Ciências Biológicas & $100 \%$ & $100 \%$ & $0 \%$ & $0 \%$ \\
\hline Engenharias & $100 \%$ & $100 \%$ & $0 \%$ & $0 \%$ \\
\hline Ciências da Saúde & $94 \%$ & $94 \%$ & $16 \%$ & $29 \%$ \\
\hline Ciências Agrárias & $100 \%$ & $100 \%$ & $3 \%$ & $8 \%$ \\
\hline Ciências Sociais Aplicadas & $16 \%$ & $52 \%$ & $35 \%$ & $58 \%$ \\
\hline Ciências Humanas & $45 \%$ & $82 \%$ & $9 \%$ & $18 \%$ \\
\hline Linguística, Letras e Artes & $7 \%$ & $40 \%$ & $10 \%$ & $18 \%$ \\
\hline Multidisciplinar & $78 \%$ & $75 \%$ & $6 \%$ & $6 \%$ \\
\hline Média geral & $\mathbf{7 1 \%}$ & $\mathbf{8 2 \%}$ & $\mathbf{9 \%}$ & $\mathbf{1 6 \%}$ \\
\hline
\end{tabular}

Fonte: Dados da pesquisa (2018).

Além de quase a totalidade de periódicos indexados na WoS e Scopus, o núcleo de Ciências da Saúde também registrou significativa indexação no SciELO e Redalyc. Essa tendência vai ao encontro do resultado obtido na Tabela 4, de uso significativo de periódicos nacionais, notadamente em função da tradição no Brasil em publicações cientificas da área de saúde.

Tabela 6 - Classificação Qualis dos periódicos do núcleo das teses da UFSC, segundo a grande área do conhecimento

\begin{tabular}{l|c|c|c|c|c|c|c|c}
\hline & A1 & A2 & B1 & B2 & B3 & B4 & C & N/A \\
\hline $\begin{array}{l}\text { Ciências Exatas e da } \\
\text { Terra }\end{array}$ & $38 \%$ & $38 \%$ & $22 \%$ & $2 \%$ & $0 \%$ & $0 \%$ & $0 \%$ & $0 \%$ \\
\hline Ciências Biológicas & $51 \%$ & $19 \%$ & $22 \%$ & $4 \%$ & $0 \%$ & $0 \%$ & $1 \%$ & $3 \%$ \\
\hline Engenharias & $90 \%$ & $10 \%$ & $0 \%$ & $0 \%$ & $0 \%$ & $0 \%$ & $0 \%$ & $0 \%$ \\
\hline Ciências da Saúde & $63 \%$ & $20 \%$ & $7 \%$ & $7 \%$ & $3 \%$ & $0 \%$ & $0 \%$ & $0 \%$ \\
\hline Ciências Agrárias & $62 \%$ & $19 \%$ & $19 \%$ & $0 \%$ & $0 \%$ & $0 \%$ & $0 \%$ & $0 \%$ \\
\hline Ciências Sociais Aplicadas & $36 \%$ & $23 \%$ & $32 \%$ & $3 \%$ & $0 \%$ & $0 \%$ & $0 \%$ & $6 \%$ \\
\hline Ciências Humanas & $36 \%$ & $18 \%$ & $0 \%$ & $9 \%$ & $9 \%$ & $0 \%$ & $0 \%$ & $28 \%$ \\
\hline Linguística, Letras e Artes & $18 \%$ & $11 \%$ & $32 \%$ & $7 \%$ & $0 \%$ & $4 \%$ & $7 \%$ & $21 \%$ \\
\hline Multidisciplinar & $74 \%$ & $23 \%$ & $3 \%$ & $0 \%$ & $0 \%$ & $0 \%$ & $0 \%$ & $0 \%$ \\
\hline Média Geral & $52 \%$ & $20 \%$ & $15 \%$ & $3,5 \%$ & $1 \%$ & $0,4 \%$ & $0,8 \%$ & $6,5 \%$ \\
\hline
\end{tabular}

A Tabela 6 mostra que mais da metade dos periódicos citados são classificados no estrato A1 e mais de $70 \%$ nos dois estratos A, demostrando uma tendência de uso de periódicos de excelência internacional por parte dos pesquisadores doutorais. As áreas com maior frequência de uso de periódicos 
A1 são Engenharias, Multidisciplinar e Ciências da Saúde e Agrárias. As áreas com maior uso de periódicos dos estratos B1 e B2 são Ciências Sociais, Humanas e Linguísticas, Letras e Artes.

Constata-se também que Ciências Humanas e Linguísticas, Letras e Artes são as áreas do conhecimento que utilizam o maior percentual de periódicos não avaliados pelo sistema Qualis, fenômeno que pode indicar um uso de periódicos nos quais ainda não há publicação de artigos por pesquisadores brasileiros.

\section{Considerações finais}

Verificou-se uma elevada média geral de disponibilidade no Portal Periódicos CAPES dos periódicos eletrônicos com artigos citados nas teses analisadas, tanto na avaliação por título, quanto na avaliação proporcional por frequência de citação de artigos. Esses índices refletem um aumento quantitativo do acervo em comparação com os resultados de pesquisas semelhantes já realizadas. Constatou-se que o aumento verificado é proporcional ao aumento do número de periódicos do acervo nos últimos cinco anos.

Sob este prisma, é possível afirmar que o acervo do Portal atende significativamente as necessidades dos pesquisadores doutorais da instituição objeto de análise e se constitui no principal meio de acesso à informação científica no Brasil. Deve, portanto, ter seu acervo mantido e até ampliado pela CAPES, não sendo alvo de restrições orçamentárias, fato que poderia prejudicar a realização de pesquisas de pós-graduação e a formação de pesquisadores e, consequentemente, o desenvolvimento da própria ciência no país.

Da análise dos periódicos com maior frequência de citação nas teses, foi possível observar que a tendência de uso prioritário deste tipo de publicação está relacionada com a citação de trabalhos publicados em periódicos internacionais indexados na WoS e Scopus e classificação Qualis dos estratos A. Esse resultado indica que o maior uso de periódicos decorre da própria influência que eles meio exercem na produção e comunicação científica em determinas áreas, 
especialmente Ciências Exatas, Biológicas, Engenharias, Ciências da Saúde e Multidisciplinar.

Por outro lado, o perfil heterogêneo dos periódicos utilizados nas Ciências Sociais, Humanas e Linguística, Letras e Artes demostram que esse tipo de publicação não deve ser utilizado como meio único de avaliação, pois seu uso não reflete com precisão os processos de produção e comunicação científica dessas áreas.

A ênfase dada ao estudo possibilitou uma visão geral das diferenças entre as grandes áreas no que tange as variáveis analisadas. Por outro lado, considerando o método adotado e a grande quantidade de teses publicadas no ano analisado, não foi possível conhecer de forma mais detalhada as características de cada grande área; limitação que pode ser suprida em estudo futuro.

Recomenda-se, por fim, a realização de pesquisas semelhantes em outras universidades federais do país, a fim de comparação dos resultados e identificação de diferenças regionais. Reforça-se também a sugestão de adoção de um formato padronizado legível por gerenciadores bibliográficos para as listas de referências das teses dos programas de pós-graduação das instituições federais de ensino, visando facilitar estudos de análise de citação, revisão sistemática e integrativa e gestão de dados de pesquisa.

\section{Referências}

BAILÓN-MORENO, Rafael et al. Bibliometric laws: Empirical flaws of fit. Scientometrics, Dordrecht, v. 63, n. 2, p. 209-229, 2005. Disponível em: https://link.springer.com/article/10.1007/s11192-005-0211-5. Acesso em: 10 fev. 2018.

BECKER, Deborah. A.; CHIWARE, Elisha. R. T. Citation Analysis of Masters' Theses and Doctoral Dissertations: Balancing Library Collections With Students' Research Information Needs. The Journal of Academic Librarianship, v. 41, n. 5, p. 613-620, 2015. Disponível em: https://www.sciencedirect.com/science/article/pii/S0099133315001226. Acesso em: 10 fev. 2018.

CANTO, Fabio Lorensi do; PINTO, Adilson Luiz. Avaliação do acervo do Portal Capes por meio da análise das citações de teses da Universidade Federal 
de Santa Catarina. In: ENCONTRO BRASILEIRO DE BIBLIOMETRIA E CIENTOMETRIA, 6., 2018, Rio de Janeiro. Anais... Rio de Janeiro, 2018. Disponível em: https://ebbc.inf.br/ebbc6/index.php/anais. Acesso em: 28 dez. 2018.

CAPES. Portal de Periódicos CAPES. Disponível em: www.periodicos.capes.gov.br/. Acesso em: 10 fev. 2018.

COUTINHO, Eliana. Aplicação da lei de Bradford à literatura técnica sobre ferrovia: análise de periódicos e avaliação da base de dados da Rede Ferroviária Federal S.A.. Ciência da Informação, v. 20, n. 2, p. 169-180, ago. 1991. Disponível em: http://revista.ibict.br/ciinf/article/view/353. Acesso em: 10 fev. 2018.

CRONIN, Blaise. The citation process: the role and significance of citation in scientific communication. London: Taylor Graham, 1984.

ENGER, K. Brock. Using citation analysis to develop core book collections in academic libraries. Library and Information Science Research, v. 31, n. 2, p. 107-112, 2009. Disponível em:

https://www.sciencedirect.com/science/article/pii/S0740818809000085. Acesso em: 10 fev. 2018.

FERNANDES, Wesley Rodrigues; CENDÓN, Beatriz Valadares. Portal de Periódicos da Capes: proposta de um modelo de estudo bibliometrico para avaliação da coleção. Revista Brasileira de Pós-Graduação, v. 7, n. 13, p. 318, 2010. Disponível em:

http://ojs.rbpg.capes.gov.br/index.php/rbpg/article/view/196. Acesso em: 10 fev. 2018.

FEYEREISEN, Pierre; SPOIDEN, Anne. Can Local Citation Analysis of Master's and Doctoral Theses Help Decision-Making about the Management of the Collection of Periodicals? A Case Study in Psychology and Education Sciences. Journal of Academic Librarianship, v. 35, n. 6, p. 514-522, 2009. Disponível em:

https://www.sciencedirect.com/science/article/pii/S0099133309001608. Acesso em: 10 fev. 2018.

FONSECA, Edson Nery. Bibliografia estatística e bibliometria: uma reivindicação de prioridades. Ciência da Informação, v. 2, n.1, p. 5-7, 1973. Disponível em: http://revista.ibict.br/ciinf/article/viewFile/19/19. Acesso em 10 set. 2018.

GALDENCIO, Roberta Cristina Barboza; LIMA, Marcia Heloisa Tavares de Figuereido. Uso de coleções periódicas por uma comunidade acadêmica da área biomédica: uma perspectiva quantitativa para o estudo de uso e gestão de coleções. Encontro Nacional de Pesquisa em Ciência da Informação, v. 16, 2015. Disponível em: 
http://www.brapci.inf.br/index.php/article/view/0000017632/ecba873d26f51710 52c98ba5b60c901d. Acesso em: 10 set. 2018.

GARFIELD, Eugene. Citation analysis as a tool in journal evaluation: journals can be ranked by frequency and impact of citations for science policy studies. Science, Washington, v. 178, n. 4060, p. 471-479, nov. 1972. Disponível em: http://science.sciencemag.org/content/178/4060/471. Acesso em 10 fev. 2018.

GIBROWSKI, Cristina. Periódicos em ciências agrárias: análise bibliométrica utilizando o article influence score do institute for scientific information.

Ciência da Informação, v. 40, n. 2, 2011. Disponível em: http://www.brapci.inf.br/v/a/21031. Acesso em: 10 fev. 2018.

GUEDES, Vania V.; BORSCHIVER, Suzana. Bibliometria: uma ferramenta estatística para a gestão da informação e do conhecimento, em sistemas de informação, de comunicação e de avaliação científica e tecnológica. In: ENCONTRO NACIONAL DE CIÊNCIAS DA INFORMAÇÃO, 6., 2005, Salvador. Anais... Salvador: ENANCIB, 2005. Disponível em: http://www.cinform-anteriores.ufba.br/vi_anais/docs/VaniaLSGuedes.pdf. Acesso em 10 fev. 2018.

HOFFMANN, Kristin.; DOUCETTE, Lise. A Review of Citation Analysis Methodologies for Collection Management. College \& Research Libraries, v. 74, n. 4, p. 321-335, 2012. Disponível em:

<http://crl.acrl.org/index.php/crl/article/view/16239/17685>. Acesso em: 02 set. 2018.

HOU, Jianhua. Exploration into the evolution and historical roots of citation analysis by referenced publication year spectroscopy. Scientometrics, Dordrecht, v. 110, n. 3, p. 1437-1452, 2017. Disponível em: https://link.springer.com/article/10.1007/s11192-016-2206-9. Acesso em: 02 set. 2018.

KAPLAN, Norman. The norms of citation behaviour: prolegomena to the footnote. American Documentation, v. 16, n. 3, p. 179-84, 1965. Disponível em: http://onlinelibrary.wiley.com/doi/10.1002/asi.5090160305/abstract. Acesso em: 02 set. 2018.

KLAES, Rejane Raffo. Indicadores bibliométricos e avaliação: a coleção de odontologia no portal de periódicos da CAPES. Encontro Brasileiro de Bibliometria e Cientometria, v. 3, p. 26, 2012. Disponível em: http://www.brapci.inf.br/index.php/article/view/0000027880/466eedf000ec6380 26fe247298346785. Acesso em: 02 set. 2018.

KUMAR, H. Anil; DORA, Mallikarjun. Citation analysis of doctoral dissertations at IIMA: A review of the local use of journals. Library Collections, Acquisitions and Technical Services, v. 35, n. 1, p. 32-39, 2011. Disponível em: 
http://www.sciencedirect.com/science/article/pii/S1464905511000108. Acesso em: 02 set. 2018.

KUSHKOWSKI, Jeffrey D. et al. Master's and Doctoral Thesis Citations: Analysis and Trends of a Longitudinal Study. Portal: Libraries and the Academy, v. 3, n. 3, p. 459-479, 2003. Disponível em:

http://lib.dr.iastate.edu/cgi/viewcontent.cgi?article=1032\&context=refinst_pub. Acesso em: 02 set. 2018.

LEYDESDORFF, Loet. Theories of citation? Scientometrics, v. 43, n. 1, p. 525, 1998. Disponível em: https://www.leydesdorff.net/citation/. Acesso em: 02 set. 2018.

MATTOS, Ana Maria; DIAS, Eduardo Wense Periódicos eletrônicos sobre administração disponíveis no portal de periódicos da coordenação de aperfeiçoamento de pessoal de nível superior: uma avaliação. Ciência da Informação, v. 39, n. 1, p. 51-66, 2010. Disponível em: http://www.brapci.inf.br/v/a/9448. Acesso em: 02 set. 2018.

MIRANDA, Ana Claudia Carvalho de Miranda; CARVALHO, Andrea Vasconcelos.; RAMOS, Anatália Saraiva Martins. Portal de periódicos da CAPES: uma política pública de acesso à informação científica e tecnológica. Biblionline, v. 11, n. 1, p. 185-204, 2015. Disponível em: http://www.brapci.ufpr.br/brapci/v/a/19463. Acesso em: 02 set. 2018.

PINTO, Adilson Luiz; ELIAS, Ezmir. Dippe; VIANNA, William Barbosa. Requisitos para métricas em arquivos: critérios específicos para arquivometria. Perspectivas em Ciência da Informação, Belo Horizonte, v.19, n.3, p.134-148, jul./set. 2014. Disponível em:

http://portaldeperiodicos.eci.ufmg.br/index.php/pci/article/view/1726. Acesso em 02 set. 2018.

RIVIERA, Emanuela. Scientific communities as autopoietic systems: the reproductive function of citations. Journal of the American Society for Information Science and Technology, v. 64, n. 7, p. 1442-1453, 2013. Disponível em: http://onlinelibrary.wiley.com/doi/10.1002/asi.22826/abstract. Acesso em: 02 set. 2018.

ROSENBERG, Zila. Citation Analysis of M.A. Theses and Ph.D. Dissertations in Sociology and Anthropology. The Journal of Academic Librarianship, v. 41, n. 5, p. 680-688, 2015. Disponível em: https://www.sciencedirect.com/science/article/pii/S0099133315001007. Acesso em: 02 set. 2018.

SANTANA, Jaciane Freire et al. Public policies for access to scientific information: periodicals portal of CAPES. Encontros Bibli: Revista Eletrônica de Biblioteconomia e Ciência da Informação, v. 17, n. 35, 2012. Disponível em: http://www.brapci.ufpr.br/brapci/v/a/12663. Acesso em: 02 set. 2018. 
SILVA, José Aparecido.; BIANCHI, Maria de Lourdes Pires. Cientometria: a métrica da ciência. Paidéia, Ribeirão Preto , v. 11, n. 21, p. 5-10, 2001. Disponível em: http://www.scielo.br/scielo.php?script=sci_arttext\&pid=S0103863X2001000200002. Acesso em: 02 set. 2018.

SILVEIRA, Murilo Artur Araújo. Produção e distinção no domínio da organização e representação do conhecimento no Brasil. Tese (Doutorado em Comunicação e Informação) - Programa de Pós-Graduação em Comunicação e Informação, Universidade Federal do Rio Grande do Sul, Porto Alegre, 2016. Disponível em: https://www.lume.ufrgs.br/handle/10183/153353. Acesso em: 20 ago. 2018.

SMALL, Henry. On the shoulders of Robert Merton: towards a normative theory of citation. Scientometrics, v. 60, n. 1, p. 71-79, 2004. Disponível em: https://link.springer.com/article/10.1023/B:SCIE.0000027310.68393.bc. Acesso em: 02 set. 2018.

SPINAK, Ernesto. Indicadores cienciometricos. Ciência da Informação, Brasília, v. 27, n. 2, p. 141-148, maio/ago. 1998. Disponível em: http://revista.ibict.br/ciinf/article/view/795/826. Acesso em: 02 set. 2018.

STRADER, C. Rockelle. Citation Analysis Do Age and Types of Materials Cited Correlate with Availability of Appropriate Library of Congress Subject Headings? Library Resources \& Technical Services, v. 56, n. 4, p. 238-253, 2012. Disponível em:

https://journals.ala.org/index.php/lrts/article/view/5076/6150. Acesso em 02 set. 2018.

TAGUE-SUTCLIFFE, Jean. Introduction to informetrics. Information Processing and Management, v. 28, n.1, p. 1-3, 1992. Disponível em: https://dl.acm.org/citation.cfm?id=160642. Acesso em: 10 fev. 2017.

VANTI, Nadia. Da bibliometria à webometria: uma exploração conceitual dos mecanismos utilizados para medir o registro da informação e a difusão do conhecimento. Ciência da Informação, Brasília, v. 31, n. 2, p. 152-162, 2002. Disponível em: http://www.scielo.br/scielo.php?pid=S0100-

19652002000200016\&script=sci_abstract\&tlng=pt. Acesso em: 25 Nov. 2018.

WALTMAN, Ludo. A review of the literature on citation impact indicators. Journal of Informetrics, v. 10, n. 2, p. 365-391, 2016. Disponível em: http://www.sciencedirect.com/science/article/pii/S1751157715300900. Acesso em: 02 set. 2018 .

WORMELL, Irene. Informetria: explorando bases de dados como instrumentos de analise. Ciência da Informação, Brasilia, D.F., v. 27, n. 2, p. 210-216, maio/ago. 1998. Disponível em:

http://www.scielo.br/scielo.php?script=sci_arttext\&pid=S010019651998000200016. Acesso em: 02 set. 2018. 


\title{
Evaluation of the Portal CAPES collection through the citation analysis of the Ph.D dissertations of the Federal University of Santa Catarina (Brazil)
}

\begin{abstract}
This work aims to evaluate the collection of the brazilian scientific data base "Portal de Periódicos CAPES" through the journals cited in Ph.D dissertations. It is also intended to analyze the most cited journals (core), identifying the country of the publications, which one are indexing in the Web of Science, Scopus, Latindex and SciELO databases and identify the Qualis/Capes classification, the brazilian system to evaluate scientific journals. All analyzes will be performed comparatively from the nine major areas of knowledge of Capes, aiming to detect similarities and differences between the patterns of use of journals and the informational needs of the Ph.D researchers. It uses the simple and random stratified sampling techniques to define the sample of $90 \mathrm{Ph} . \mathrm{D}$ dissertations, 10 of each major area of knowledge, accounting for $20.3 \%$ of the 443 dissertations presented in 2015 at the Federal University of Santa Catarina (Brazil). The results indicate that $84.76 \%$ of the electronic journals cited in the dissertations are available in Portal de Periódicos CAPES. Biological Sciences $(94.15 \%)$ is the highest availability area and Linguistics, Arts and Letters (69.23\%), the lowest . International journals, mainly from the United States, England and the Netherlands, are most used in most areas, except in the Social Sciences, Humanities and Linguistics, Letters and Arts, which use more brazilian journals. It is found, therefore, that the Capes Journals portal is significantly adequate to the needs of $\mathrm{Ph} . \mathrm{D}$ researchers, especially in the technological, natural and health sciences, wich uses manly international journals, indexed in the Web of Science and Scopus and classify at A1 and A2 Qualis/Capes.
\end{abstract}

Keywords: Scientific journals. Citation analysis. Metric studies of information. Portal de Periódicos Capes.

Recebido: 24/09/2018 Aceito: 03/12/2018

\footnotetext{
${ }^{1}$ Programa de Biblioteca Eletrônica, criado em 1999 por meio de um consórcio mantido pela Universidade de São Paulo (USP), Universidade Estadual Paulista (UNESP), Universidade Estadual de Campinas (UNICAMP), Universidade Federal de São Carlos (UFSCAR), Universidade Federal de São Paulo (UNIFESPE) e apoio da Fundação de Amparo à Pesquisa do Estado de São Paulo (FAPESP).
} 